\title{
Oddity of visual patterns conceptualized by pigeons
}

\author{
CELIA M. LOMBARDI, CARLOS C. FACHINELLI, and JUAN D. DELIUS \\ Psychologisches Institut, Ruhr-Universität, Bochum, West Germany
}

\begin{abstract}
Pigeons were trained to learn an instrumental oddity-from-sample discrimination involving visual forms. One group, the "few examples" group, dealt with 5 patterns in 40 different combinations. Another group, the "many examples" group, dealt with 20 patterns in 160 different combinations. After both groups had reached asymptotic performance and had learned to operate under partial reinforcement conditions, they were tested for transfer under extinction conditions with two different groups of 5 novel patterns, each in $\mathbf{4 0}$ combinations. All animals showed significant above chance transfer to both of these novel stimulus sets. Transfer performance with test stimuli of similar geometric design to training stimuli was better than performance with stimuli of markedly different design. The transfer performance of the "many examples" group was marginally better than that of the "few examples" group, even though the latter's performance on the training stimuli was better throughout. It is concluded that pigeons can learn to employ an oddity concept and that this may be promoted by the use of many training exemplars. Furthermore, it is inferred that pigeons may normally use a mixture of strategies to solve oddity and identity problems.
\end{abstract}

The matching-to-sample discrimination paradigm, in which subjects must decide which of two comparison stimuli is identical with a previously presented sample stimulus, has been extensively used with pigeons since Cumming and Berryman (1961) developed an efficient version for use with this species. But there still seems to be doubt about whether pigeons can learn the general principles of identity and oddity that are conducive to an efficient mastery of the task. Carter and Werner (1978) reviewed the evidence extant up to 1978 and decided that pigeons did not learn identity/oddity concepts, but rather were conditioned to apply discrete rules of a stimulus-response type. Soon afterwards, Zentall and Hogan (1978) came to the opposite conclusion. The empirical evidence that has accrued since then has been equivocal, sometimes supporting one position, sometimes the other (e.g., Holmes, 1979; Wright \& Sands, 1981; Zentall, Edwards, Moore, \& Hogan, 1981). Two recent reviewers (Mackintosh, 1983; Macphail, 1982) accordingly reached confliciting, but reserved, opinions about pigeons' capabilities in this matter.

This research was supported by the Deutsche Forschungsgemeinschaft through its Sonderforschungsbereich 114 and the Research Fund of the Ruhr-Universität. In addition, C. M. Lombardi received a grant from the Deutscher Akademischer Austauschdienst, and C. C. Fachinelli received a travel grant from the Heinrich Hertz Stiftung. C. C. Fachinelli's permanent address is: Instituto de Psicología, Universidad del Aconcagua, Mendoza, Argentina. We are grateful to V. Hollard, E. Curio, G. Keim, P. Kulik, A. Lohmann, D. Hagenkötter, and Julia Delius for their cooperation and assistance. Reprint requests should be addressed to J. D. Delius, Experimentelle Tierpsychologie, Psychologisches Institut, Ruhr-Universität, D 4630 Bochum, West Germany.
During research into the rotational invariance of pattern recognition by pigeons using the matching-tosample method, we obtained some incidental, but nonetheless strong, support for the notion that pigeons are indeed capable of using a generalized identity/oddity rule (Hollard \& Delius, 1982). The animals had shown immediate matching-to-sample performance transfer to novel geometrical patterns. Furthermore, we were impressed by the fact that an animal like the pigeon must in its normal environment necessarily make a multitude of decisions about the equivalence or nonequivalence of a variety of percepts if it is to survive and reproduce. It would be very uneconomical in terms of information processing if the pigeon did not have command over a universal procedure that it could apply in all such situations. At least some of the research cited by the reviewers mentioned above as having yielded evidence contrary to this notion can be criticized for having employed tasks that indeed invited the subjects to more simple solutions without recourse to ruies of a concept type or for not having taken sufficiently into account the interference that can arise from the pigeon's strong novelty aversion.

Since we were interested in continuing to use the matching-to-sample (or rather its analogous converse, the oddity-from-sample procedure), we undertook an experiment to clarify the situation. It was based on the appraisal that the best way to demonstrate the operation of a concept-like rule is to show that animals can generalize it to a novel set of stimuli about which they have not previously learned anything and about which they cannot learn any specific rules during testing because they experience them only under extinction conditions. We also varied the number of examples used during 
training; a concept is learned on the basis of examples, and it seems plausible that their number is a potentially important factor for concept development.

\section{METHOD}

\section{Subjects}

Ten adult homing pigeons (Columba livia) of local origin were used. They were housed in individual cages located in a well-ventilated room, kept at $18^{\circ} \mathrm{C}$, with a 14-h light, 10-h dark cycle. The birds were maintained at $80 \%$ of their normal weights throughout the experiment.

\section{Apparatus}

A three-key Skinner box of conventional design was employed. The stimuli were back-projected onto the response keys with the aid of an automatic projector. Three electromagnetic shutters placed directly behind the keys separately controlled the displays. The projector was equipped with photocells that sensed the presence or absence of coding perforations in specially made slide frames. A group of 30 patterns were drawn by a draftsman, who modified five basic designs six times over. A further group of 10 patterns were drawn without reference to the above group by an art student. All the patterns were drawn in black ink on white paper. Photographically reduced negatives were affixed to the slide frames. The stimuli, one to a key, appeared as white patterns of about $10 \times 10 \mathrm{~mm}$ on the dark background of the 25-mm-diam keys. A microcomputer controlled all events within the experimental sessions and recorded with a printer the relevant performance data on a trial by trial basis.

\section{Procedure}

The pigeons were autoshaped to peck the illuminated middle key for several sessions with food access as reward. A few birds that still did not respond were shaped by hand in further sessions. Then the animals were trained to peck the three keys as these were singly illuminated in random order. During the last two sessions of this phase, a white shape on a black background was projected on the keys. After a total of 11 pretraining sessions, one subject that did not respond regularly was excluded. The remaining nine pigeons began with the oddity-from-sample discrimination training.

Training. A trial began with a pattern, the sample stimulus, being projected on the middle key. As soon as the subject pecked this key, the two comparison stimuli, one of which had a pattern identical to that shown on the middle key and the other of which had a different pattern, were separately projected on the two side keys. If the animal responded to the latter, odd pattern, the stimuli on all three keys were extinguished, grain was offered for $3 \mathrm{sec}$, and a reinforcement light came on. If the subject chose the key bearing the identical pattern, the pigeons were punished with a 3-sec time-out with both the stimuli and the houselight extinguished. The houselight was otherwise on throughout the session. The time that elapsed between the onset of the comparison stimuli and the response to a side key was recorded as reaction time. The next trial began $8 \mathrm{sec}$ (in early sessions $12 \mathrm{sec}$ ) later. If the subject had responded incorrectly in the last trial, the new trial was a simple repetition, a correction trial in which the same patterns were presented on the same keys. If the pigeon had responded correctly during the last trial, the pattern combination shown in the next was chosen randomly from the group of sets in use, with the restriction that the position of the odd pattern on the left or right side key was determined by a quasi-random sequence (Fellows, 1967). Only the outcome of noncorrection, fair trials was used for performance evaluation. A session consisted of 40 such trials, and the sessions were run daily.
The pigeons were divided in two groups of five and four subjects each, matched according to their performance during the pretraining phase. The smaller group was trained with three-stimulus (one-sample, two-comparison) sets constructed on the basis of five different patterns (see Figure 1). Any one sample pattern could be combined with four different odd comparison patterns, the identical comparison pattern of course being always the same as the sample. Thus, there were 20 different combinations. Each combination occurred in two versions: one with the odd comparison appearing on the right side key, the other with it appearing on the left side key, thus generating the $\mathbf{4 0}$ sets shown in each session.

The other, larger group of pigeons dealt with three-stimulus sets based on 20 patterns (see Figure 1). There were 380 possible combinations. Since this was an excessive number to manage, we chose 80 of them at random with the constraint that each of the 20 patterns appear equally often and that the odd comparisons be equally often on the right and on the left. Again because of the odd-left and odd-right versions of each combination, this yielded 160 stimulus sets. They were divided at random, with the same constraints as given above, into four collections of 40 sets, and these were used one per session in a rotating fashion.

In the course of the first sessions the subjects were required to respond an increasing number of times to the sample patterns on the center key. From Session 7 onwards, they had to peck it 15 times before the comparison patterns appeared on the side keys. After having to deliver two consecutive responses on the side keys during Session 49, from Session 50 onwards, the subjects had to peck five times in an unbroken sequence on such a key to obtain reinforcement or punishment. Reaction times were now measured to the first response on a side key. Correction trials were no longer required. Partial reinforcement was introduced soon afterwards, and from Session 56 onwards, only half of the trials yielded reward or time-out according to Fellows's (1967) sequences. The remainder led directly to the intertrial interval.

Testing. The training phase ended with the 57 th session, and the testing phase began forthwith. The testing phase consisted of two series of 10 consecutive sessions. Each session within these series incorporated 24 trials that were fully equivalent to the final ones of the training phase, as described above. The remaining 16 trials were transfer trials that were inserted in a randomized fashion among the training trials of each session, avoiding, however, the first three and the last three trials. Eight of these trials, the novelty habituation trials, involved the presentation of stimulus sets constructed from one series of five novel patterns, the terminal responses to which were consistently reinforced or punished. The other 8 trials, the true transfer trials, involved stimulus sets constructed from another, different series of five novel patterns. However, the terminal responses to these test sets were never reinforced, neither positively nor negatively, but led directly to the intertrial interval.

For the first series, each of the two collections of transfer sets was constructed from two different groups of five patterns that were unknown to the subjects but similar in geometrical style to those used for training (see Figure 1, but note that only the patterns used in the nonreinforced test are illustrated). As explained earlier, five patterns yield a total of 40 stimulus sets. Each of these sets was accordingly shown twice over the 10 transfer sessions, interspersed among the training combinations, as explained.

As soon as the first series of transfer sessions was completed, a second series was run according to the same procedure but using two new collections of stimulus sets, each based on two different groups of five novel patterns that were of a geometrical style markedly different from that characterizing the training stimuli and the transfer stimuli of the previous series (see Figure 1 , but note that only nonreinforced test patterns are shown and that the reinforced patterns were distinctly different). 
The purpose of including reinforced novel stimulus sets was to prevent the subjects from associating novelty with nonreinforcement. The sets intended to test for oddity transfer were the novel nonreinforced ones. The subjects could obviously not learn any specific rules about these latter sets throughout the testing sessions.

\section{RESULTS}

The performance of each individual on the training component was evaluated in terms of the percentage correct responses produced during each session. During the test phase, an analogous index was also calculated separately for the two kinds of test components. Mean performance scores were calculated for each session and separately for the two groups of birds, the "few examples" group and the "many examples" group. These are plotted as a function of the succession of sessions, with some abbreviations, in Figure 1. It can be seen that the "many examples" group was somewhat slower than the "few examples" group in acquiring the oddity-fromsample performance, and that it did not reach the same asymptotic level of performance. But the difference is not significant (Mann-Whitney $U$ test based on the 10 last training sessions, $\mathrm{p}>.05$ ).

For the sessions incorporating the transfer tests, Figure 1 also illustrates performance on the novel stimulus sets shown under extinction conditions throughout, that is, without any feedback about the correctness or incorrectness of responses. Because there were 8 such test trials per session, as compared with 24 training trials, the means shown are based on pairs of sessions, that is, on 16 test trials, to make them more nearly comparable in terms of variance. It is apparent that the transfer to these stimuli was well above the chance, $50 \%$, score level. Separate statistical analyses confirm this (binomial tests, each subject in each test series, $\mathrm{p}<.05$ or better; combined $\mathrm{p}<.001$ ). However, it is also clear that performance on the test stimuli did not reach the level concurrently achieved on the training stimuli. Also, the mean reaction times to the test stimuli were significantly slower than those corresponding to the training stimuli (the "many" and "few examples" groups were pooled because they did not differ; Test Series $1,1.01 \mathrm{sec}$ vs. $0.86 \mathrm{sec}$; Test Series $2,1.51 \mathrm{sec}$ vs. $0.85 \mathrm{sec}$; Wilcoxon tests, both $\mathrm{p}<.01$ ). Overall, the "many examples" group's transfer performance seems superior to that of the "few examples" group, particularly in relation to their performance on the training components, where the reverse is the case. However, the "many examples" advantage is barely significant only in the second test series (Mann-Whitney test, $\mathrm{p}<.05$ ).

Transfer performance on the first test series involving a set of patterns of a style similar to the training stimuli but under continuous extinction was significantly superior to that shown during the second analogous series in which the patterns were of a different and more varied style ("few" and "many examples" groups pooled, Wilcoxon test, $\mathrm{p}<.01$ ). Also, the reaction times to the test sets were slower in the second series than in the first (groups pooled, $1.51 \mathrm{sec}$ vs. $1.01 \mathrm{sec}$, Wilcoxon test, $\mathrm{p}<.01$ ). It seems that performance on the habitual training stimuli also decayed somewhat, although not significantly, during the first sessions of these test series.

Figure 1 also shows a summary of the results over the final 10 training sessions and the two test series, including also the mean outcome of the trials with the two sets of novel stimuli that were consistently reinforced. The animals' performance on these sets was in every respect closely similar to that achieved with the nonreinforced test sets. This is reflected by the fact that the subjects' performance ranking on both components correlated positively (Test 1, Kendall's $\tau=+0.54, \mathrm{p}<.05$; Test 2 , Kendall's $\tau=+0.78, \mathrm{p}<.01$ ), whereas these ranks did not correlate significantly with those deriving from performance on the training stimuli in the same test series. Although this is not shown in Figure 1, there was no evidence of any performance improvement on the reinforced sets with succeeding sessions, as might perhaps have been expected inasmuch as the animals were receiving full feedback for responses to them.

\section{DISCUSSION}

Three mechanisms have been proposed by which animals can solve the oddity-from-sample (or actually the more commonly used analogous, but converse, matching. to-sample) task. They may learn a single generalized concept-like rule that is independent of the type of stimulus to which it is applied, they may learn a series of separate and sample-specific stimulus-response chains, or they may learn a set of discriminations based on the different overall stimulus configurations. The present study examined whether pigeons could apply an oddity concept-type rule, something it has been doubted they were capable of. To prove this, it is necessary to design a situation where the discrimination performance cannot be explained by the other two mechanisms consid. ered to be more parsimonious than the concept construct (Carter \& Werner, 1978).

It is agreed that neither the configuration nor the multiple-rule hypothesis can explain the transfer of discrimination to test sets constructed from stimuli that were not used during training. There is no doubt that our pigeons showed transfer when faced with the novel test sets. The nature of the stimulus newness, however, needs to be considered more closely. The patterns used to assemble the test set for the first series were similar in geometric style to those used during training. It is conceivable that, through common stimulus generalization, these stimuli were perceived as equivalent to the training stimuli, allowing the application of simple stimulus-response principles. The test sets used in the second series, however, were constructed from stimuli 

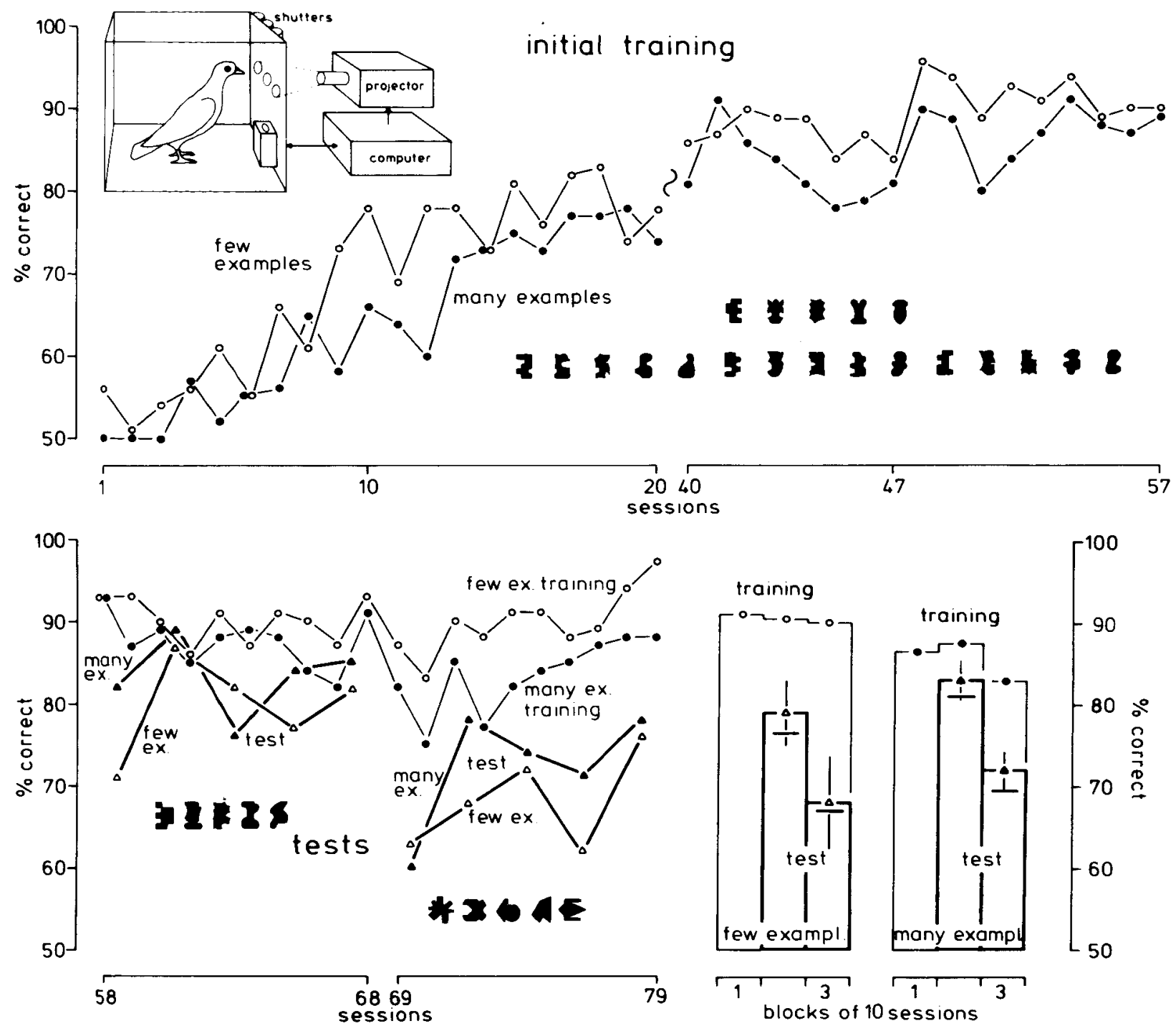

Figure 1. Top: Mean oddity-from-sample learning curves for the "few examples" and "many examples" groups of pigeons. Sessions 21 to 39 have been left out for space-saving reasons. The inserts illustrate the apparatus and the patterns used for training. Only the upper 5 training stimuli were used for the "few examples" group. All 20 pattems were used for the "many examples" group. Bottom: Performance of the same pigeon groups in the transfer test sessions. Left: Session-by-session performance on the training and the nonreinforced test components. To approximately equalize the number of trials on which the data points are based, the test results from two consecutive sessions were combined (see text). The inserts show the nonreinforced test stimuli, which were the same for both groups of pigeons. Right: Summary for the last 10 training sessions and the 2 test session blocks of 10 sessions each. Mean performance on the training and the novel nonreinforced test stimuli (with standard deviations) are shown separately. The short horizontal bars indicate performance on the reinforced novel stimuli (not shown, see text).

markedly different in style from those used in training. Common stimulus generalization could not conceivably have helped the pigeons. The pigeons' above-chance performance with these test stimuli implies that they employed a single, concept-type rule. Moreover, the closely correlated performance of the individual pigeons on both test series (Kendall's $\tau=+0.68, p<.01$ ) suggests that they treated the transfer stimuli of both series in a similar way.

The question is now why the transfer was not complete. If the animals learned a concept-like oddity rule, performance on the test sets should have been equivalent to that on the training set. The less than optimal transfer both in performance and latency could be due 
to the notorious novelty aversion of pigeons, promoting the choice on the basis of spontaneous and irrelevant preferences. Alternatively, it may be that familiarity with specific stimuli during training promotes the memorability of the sample stimuli as the animal looks away from the center key, thus improving decisions about the oddity of comparison stimuli. Another possibility is that the pigeons only partly used an oddity concept, and supplemented it with configuration discrimination and sample-specific rules, as the three mechanisms do not necessarily exclude each other. Such a combined strategy would have enabled high-level performance with the training stimuli but not with the test stimuli when the two latter components were inapplicable. The assumption of a mixed strategy is not popular with theorists (e.g., Carter \& Werner, 1978) but may nevertheless be realistic. It is, for example, well established that, when homing, pigeons bring to bear several different orientation mechanisms in an entangled and flexible manner (Papi \& Wallraf, 1982). Pigeons may also be nonpurists in the present context, and use different strategy mixes in different situations or according to individual propensities. This might explain the discordance among earlier studies on whether or not oddity/identity concepts are employed by pigeons.

Although both acquisition and terminal training performance was somewhat worse in the "many examples" group than in the "few examples" group, their relative achievement with the test stimuli was marginally, though not significantly, superior. In terms of information processing parsimony, this trend seems natural. While learning about a few stimuli can be done simply, on a one by one basis, this becomes impractical with many stimuli, both because of the load on memory and because of interference between them. A general transferable principle is then more economic, even though initially it may require more complex processing.

Most previous studies on identity/oddity in the pigeon have used relatively few training exemplars, and thus it is not surprising that the evidence for conceptualization has been meager. We also venture the conjecture that the often used color stimuli may be inadequate to demonstrate concept formation because of their exceptional salience and memorability for pigeons (Nelson \& Wasserman, 1981) and because pigeons can generalize extensively between them (Wright \& Cumming, 1971).
In any case, it must now be incontrovertibly accepted that an abstract concept of oddity (and by implication identity) can control the behavior of pige ons in suitable situations. This is exactly what we set out to demon. strate. However, we do not believe that we taught the pigeons the concept; rather, we think they already had it before the experiment began. What we taught them was to use it in a particular situation.

\section{REFERENCES}

Carter, D. E., \& Werner, T. J. (1978). Complex learning and information processing by pigeons: A critical analysis. Journal of the Experimental Analysis of Behavior, 29, 565-601.

Cummina, W. W., \& Berryman, R. (1961). Some data on matching behavior in the pigeon. Journal of the Experimental Analysis of Behavior, 4, 281-284.

Fellows, B. J. (1967). Chance stimulus sequences for discrimination tasks. Psychological Bulletin, 67, 87-92.

Hollard, V. D., \& Delius, J. D. (1982). Rotational invariance in visual pattern recognition by pigeons and humans. Science, 218, 804-806.

Holmes, P. W. (1979). Transfer of matching performance in pigeons. Journal of the Experimental Analysis of Behavior, 31, 103-114.

MACKINTOSH, N. J. (1983). Conditioning and associative learning. Oxford: Clarendon.

MACPHAIL, E. (1982). Brain and intelligence in vertebrates. Oxford: Clarendon.

Nelson, K. R., \& Wasserman, E. A. (1981). Stimulus asymmetry in the pigeon's successive matching-to-sample performance. Bulletin of the Psychonomic Society, 18, 343-346.

Papi, F., \& Wallraf, H. G. (Eds.) (1981). Avian navigation. Berlin: Springer.

Wright, A. A., \& Cumming, W. W. (1971). Color-naming functions for the pigeon. Journal of the Experimental Analysis of Behavior, 15, 7-17.

Wright, A. A., \& SAnds, S. F. (1981). A model of detection and decision process during matching-to-sample by pigeons: Performance with 88 different wavelengths in delayed and simultaneous matching tasks. Journal of Experimental Psychology: Animal Behavior Processes, 7, 191-215.

Zentall, T. R., Edwards, C. A., Moore, B. S., \& Hogan, D. E. (1981). Identity: The basis for both matching and oddity learning in pigeons. Journal of Experimental Psychology: Animal Behavior Processes, 7, 70-86.

Zentall, T. R., \& Hogan, D. E. (1978). Same/different concept learning in the pigeon: The effect of negative instances and prior adaptation to transfer stimuli. Journal of the Experimental Analysis of Behavior, 30, 177-186.

(Manuscript received February 3, 1983; revision accepted for publication October 11, 1983.) 